\title{
Stroke code simulation lab
}

\author{
Awss Zidan*, Umair Afzal, Yi Mao, Deborah Y Bradshaw and Julius G Latorre \\ Department of Neurology, SUNY Upstate Medical University, USA
}

\begin{abstract}
Introduction: The lack of standardized training for neurology residents in the management of stroke codes allows for suboptimal care, and may negatively impact patients' outcome. We designed and implemented a stroke code simulation lab with the objective of improving door to needle time for acute stroke thrombolytic therapy.

Method: Thirteen trainees were enrolled in the simulation lab. Each participant was asked to lead a mock stroke code performed on a standardized patient. The mock cases included clinical vignettes that mimicked real-life scenarios. The effect of the simulation lab on the trainees' level of knowledge was assessed using pre- and post-course tests and a course survey. To test whether implementing a simulation lab reflected directly on patients' outcome, a 12-month retrospective chart review to compare door-to-needle time DNT before and after stroke codes was undertaken.
\end{abstract}

Results: Door-to-needle time improved by 12 minutes following the implementation of the simulation lab (52 minutes before, versus 40 minutes after. P value 0.012 ). Pre- and post-training knowledge test scores improved from $61 \%$ to $81 \%$. ( $\mathrm{p}=0.00002)$. A 6 -question survey showed that the course was received favorably by the participating trainees.

Conclusion: Stroke code simulation lab can be an effective tool in improving neurology residents' knowledge of responding to and managing stroke codes. Moreover, the simulation reduced door-to- needle time indicating its potential usefulness in improving patient outcomes.

\section{Introduction}

The establishment of hyperacute systems mobilization in the rapid evaluation and treatment of stroke using stroke codes in the hospital setting made it possible for intravenous thrombolysis to be administered within 60 minutes of patient presentation to the emergency room. Stroke codes notify all involved personnel of the arrival of stroke victims who may be candidates for therapeutic stroke interventions. It aims to increase the percentage of patients eligible for thrombolytic therapy and to shorten the delay between patients arrival and their first neurological assessment [1].

Despite the success that simulation has achieved in critical fields of medicine, such as basic and advanced cardiac life support, it has not yet been systematically utilized in the field of neurology. We have designed and implemented a stroke code simulation lab by creating mock cases that mimicked real-life stroke codes, with the objective of improving door to needle time for acute stroke thrombolytic therapy.

\section{Methods}

Design: Thirteen mock cases were created for standardized patients (SP). Each SP case included demographics (gender and age) and a clinical scenario including history of the presenting symptoms, laboratory data and radiological images (CT head with $\backslash$ without vessels imaging). Each participating resident assumed the role of the primary responder for one mock case. Seven PGY-2 and six PGY-3 underwent training through the simulation lab, while five PGY-4 residents prepared the materials and supervised the performance of the mock cases.

Performance was evaluated systematically using a standardized checklist containing discrete elements that are necessary for clinical decision making for acute stroke thrombolysis, including accurate last-known-well time, completion of the inclusionlexclusion criteria for IV-tPA, performance of full and accurate National Institute of Health Stroke Scale NIHSS. A number of mock cases also include clinical vignettes focusing on common stroke mimics and patients with previous absolute contraindications that are now considered eligible using the updated thrombolysis criteria [2]. Consider Figure 1 for a sample of a clinical scenario and a corresponding checklist.

\section{Data acquisition}

A stroke-specific knowledge test was administered pre-training and then 30 days later after completion. In addition, a course-evaluation survey was offered to all participating residents at the end of the course.

A retrospective charts review for all stroke codes in the six months preceding and the six months following the simulation course was undertaken.

Statistical Analysis: Microsoft Excel (version professional plus 2013) was used for statistical analysis. Pre- and post-course testing scores were compared using paired-t-test. The evaluation survey results were plotted on a scale from 0 (strongly disagree) to 5 (strongly agree), with 5 always representing a more positive attitude toward the course, descriptive analysis was then used.

Correspondence to: Awss Zidan, M.D., Department of Neurology, SUNY Upstate Medical University, 750 E Adams St, Syracuse, NY, 13210, Tel: 3154645357; Fax: 3154645355; E-mail: Zidana@upstate.edu

Key words: stroke code, simulation, door-to-needle, time, tpa, tissue plasminogen activator, revascularization

Received: August 24, 2017; Accepted: September 21, 2017; Published: September 25, 2017 


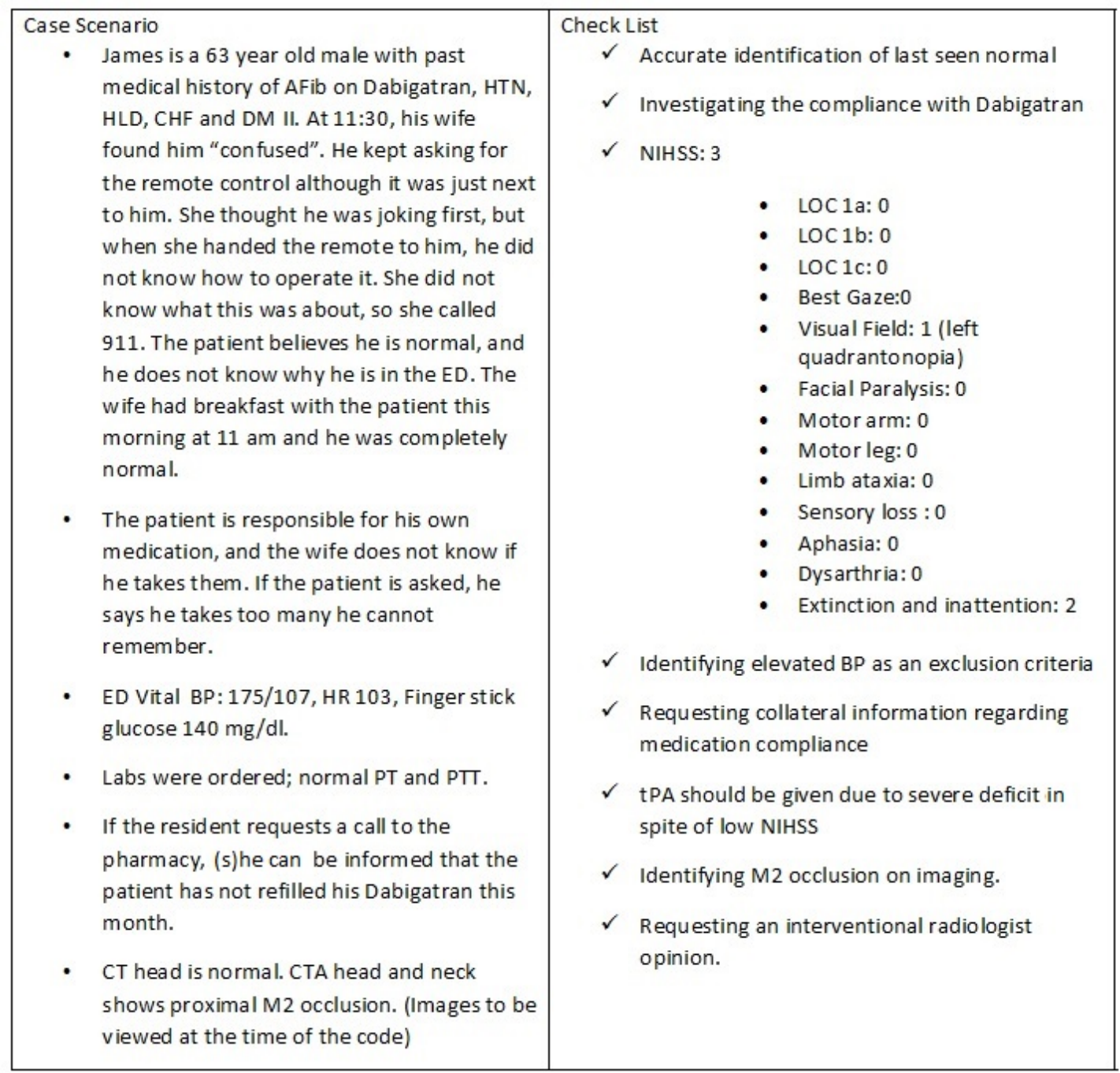

Figure 1. A sample of the clinical scenario of a mock case (left), and the checklist of the elements trainees needed to identify correctly (right)

The primary outcome of the study was improvement in Door-toNeedle time (DNT) before and after implementation of the stroke code simulation lab using the Wilcoxon rank sum test.

\section{Results}

Thirteen $(\mathrm{N}=13)$ neurology residents participated in the simulation lab. Mean residents scores on pre-training and post-training test were $61 \%$ vs $81 \%$, respectively, $p=0.00002$.

Analysis of 6-questions-survey (11 responders) showed a weighted average of 4.65 ( $95 \% \mathrm{CI}=4.51-4.79)$ on a scale from 1 to 5 , with 5 always indicating a favourable answer (Figure 2).

There were 34 stroke code activations in the preceding 6 months prior to the stroke code simulation lab. There were 41 stroke code activations in the 6 months following the stroke code simulation lab. There was a statistically significant 12 minute reduction between the mean DNT presimulation and postsimulation period (52 $\min \pm 10$ vs $40 \pm 5$, $\mathrm{p}$ value 0.012 ).

\section{Discussion}

In academic centers, stroke codes rely heavily on neurology residents who examine the patients and determine eligibility for thrombolytic therapy. The time of administration of thrombolytic therapy is the critical factor in achieving robust recovery, as there is an estimated loss of approximately two million neurons with every elapsed minute following the stroke symptom onset without reperfusion [3]. Hence, a reduction in door-to-needle time (DNT) can be a potential target to improve stroke outcome [4].

Achieving an optimal DNT following a stroke code can be hindered by many obstacles, and includes the following: provider stress, lack of experience in performing a very rapid but accurate neurological assessment and lack of knowledge of critical interpretation of the laboratory and radiological data generated during the code. A Study has shown that experienced neurologists have reduced DNT compared to novice neurologists [5]. Analyzing DNT in community hospitals demonstrate a reverse linear relationship between prior cumulative exposure to stroke cases and DNT of the treating physicians [6]. This a reason for concern, and a call for inventing new educational methods that can close the gap, and provide patients with the best possible outcome.

Our project should only serve as a first step toward developing a more comprehensive and educational simulation lab. Future projects may aim to involve multiple members of the stroke-code-response 


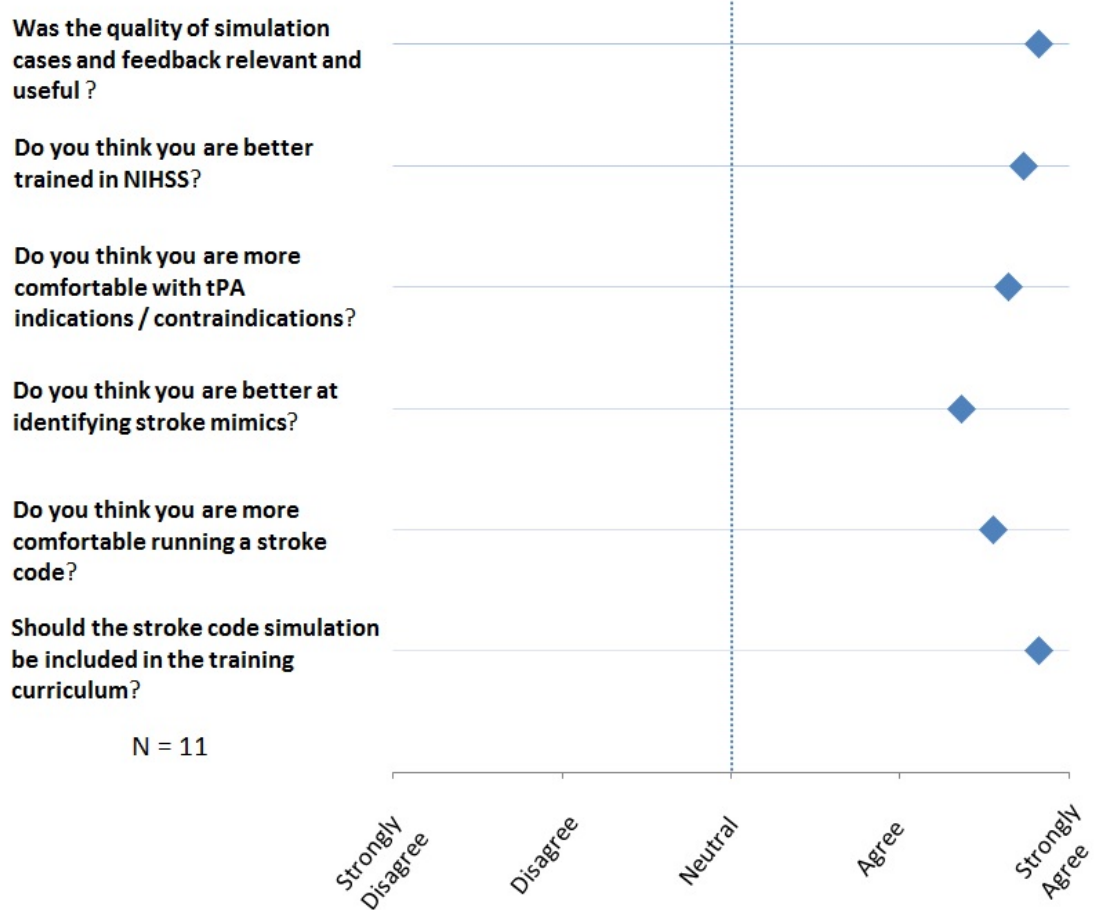

Figure 2. A plot showing results of post-course survey administered to all participating trainees. Responses were weighted on a numerical scale from 1 (strongly disagree) to 5 (strongly agree)

team such as nurses, technicians, radiologists, and ED physicians in order to allow for better communication and parallel work among the team members.

We admit to few limitations in our study. The project was activated in synchrony with other protocols that aimed to improve the stroke care quality in our institution, which limits our ability to attribute the improvement in DNT solely to the stroke code simulation lab. We also acknowledge the small number of the stroke codes and the short period of the study. On the other hand, it would be expected that the effect of the simulation lab would abolish with time, in the absence of refreshing courses. Thus, a well-designed double-arm cross-over study will be required in the future to delineate the full extent of benefit of installing stroke code simulation labs in academic centers.

\section{Conclusion}

The stroke code simulation lab was effective in meeting its objectives of improving the neurology residents' knowledge required for responding to and managing stroke codes, and it was received favorably by them. Data analysis revealed that implementing the simulation reduced door-to-needle time, proving its potential usefulness in improving patient outcome.

\section{Acknowledgment}

Stroke code simulation lab was innovated as a quality improvement project. The projects was heavily supported by Dr. Amy E Sanders, Dr. Ramesh Cherukuri, and Dr. Usman Qadeer.

\section{Author contributions}

Awss Zidan: simulation lab concept and design, and writing the manuscript; Umair Afzal: simulation lab design and supervision, and acquisition of data; Yi Mao: acquisition of data, analysis and interpretation of data; Julius G Latorre: drafting and revising the manuscript for intellectual content, analysis and interpretation of data; and Deborah Y Bradshaw: drafting and revising the manuscript for intellectual content.

\section{References}

1. Belvís R, Cocho D, Martí-Fàbregas J, Pagonabarraga J, Aleu A, et al. (2005) Benefits of a prehospital stroke code system. Cerebrovasc Dis 19: 96-101. [Crossref]

2. Demaerschalk BM, Kleindorfer DO, Adeoye OM, Demchuk AM, Fugate JE, et al (2016) Scientific Rationale for the Inclusion and Exclusion Criteria for Intravenous Alteplase in Acute Ischemic Stroke. Stroke 47(2): 581-641.

3. Saver JL (2006) Time is brain-quantified. Stroke 37: 263-266.

4. Fonarow GC, Smith EE, Saver JL, Reeves MJ, Bhatt DL, et al. (2011) Timeliness of tissue-type plasminogen activator therapy in acute ischemic stroke patient characteristics, hospital factors, and outcomes associated with door-to-needle times within 60 minutes. Circulation 123: 750-758. [Crossref]

5. Zarza B, Alonso dLM, García-Barragán N, Díaz-Sánchez M, López-Sendón JL, et al (2007) Influence of the experience and of out-of-hospital stroke code in thrombolytic treatment of acute stroke. Neurologia (Barcelona, Spain) 23: 349-355. [Crossref]

6. Chong CA (2016) Using stroke thrombolysis to describe the role of repetition in learning a cognitive skill. Medical education 50: 250-258.

Copyright: (C2017 Zidan A. This is an open-access article distributed under the terms of the Creative Commons Attribution License, which permits unrestricted use, distribution, and reproduction in any medium, provided the original author and source are credited. 